Self-assessment corner

\title{
An unusual cause of orthopnoea
}

\author{
Paul Donohoe, Walter T McNicholas
}

Department of Respiratory Medicine, University College Dublin, St. Vincent's Hospital, Elm Park,

Dublin 4, Ireland $P$ Donohoe

WT McNicholas

\section{Correspondence to} Dr McNicholas

Accepted 5 September 1995
A 67-year-old man suffered a fracture of his right clavicle and right-sided ribs in a road traffic accident. One week later he developed progressive dyspnoea, severe orthopnoea and peripheral oedema. Physical examination revealed a tachypnoea of 20 breaths/minute, and central cyanosis; other vital signs were normal. Jugular venous pressure was elevated at $4 \mathrm{~cm}$ above the sternal angle. Chest percussion revealed bibasal dullness, and auscultation revealed fine end-inspiratory crepitations. A third heart sound was audible at the left sternal border. There was evidence of sacral and lower limb oedema, up to mid-calf. Neurological examination revealed absent left biceps and supinator tendon jerks, with left sternomastoid weakness, consistent with a left 7 th cervical root lesion.

His chest X-ray is shown in the figure. Pulmonary function tests showed FEV 1.251 (36\% predicted), FVC 1.591 (36\% predicted).

Resting electrocardiogram showed normal sinus rhythm, no evidence of ischaemia and a normal axis. White cell count was $6.2 \times 10^{9} / 1$, haemoglobin $15 \mathrm{~g} / \mathrm{dl}$, and erythrocyte sedimentation rate $4 \mathrm{~mm} / \mathrm{h}$. Urea and electrolytes, liver function tests were also normal.

The initial impression was of congestive cardiac failure, and he was treated with intravenous frusemide and captopril. One week later, his signs of congestive cardiac failure had fully resolved, but his orthopnoea persisted, and was of sufficient severity to warrant him sleeping upright in a chair. An echocardiogram and multi-gated acquisition scan were attempted, but were unsuccessful due to the patient's inability to lie flat (because of severe orthopnoea). A multigated acquisition scan was repeated under sedation, together with pulse oximeter monitoring. This was associated with rapid severe oxygen desaturation to $70 \%$, and a paradoxical ribcage and abdominal breathing pattern was observed.

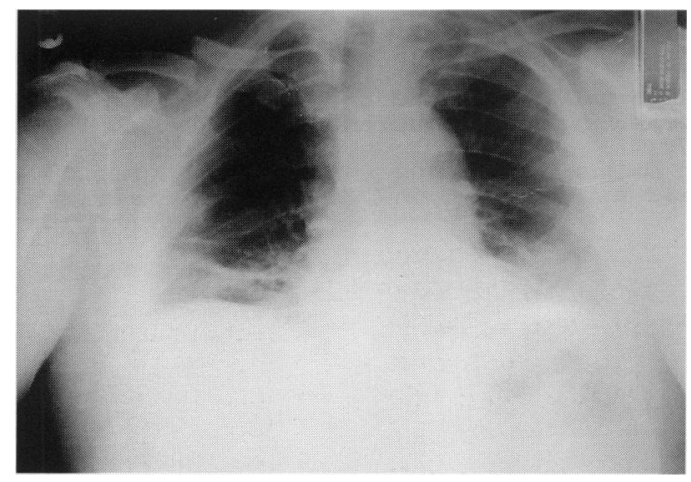

\section{Questions}

1 What is the diagnosis?

2 How may it be confirmed?

3 What are the options for treatment?

Figure 


\section{Answers}

QUESTION 1

Post-traumatic bilateral diaphragmatic palsy. The persistent orthopnoea, despite clearing of clinical signs of congestive cardiac failure, the documented desaturation on assuming the recumbent position, the paradoxical rib cage and abdominal breathing pattern noted when lying flat, the elevated hemidiaphragms on chest X-ray, the left seventh cervical root signs, are all pointers to the diagnosis.

\section{QUESTION 2}

Fluoroscopic screening of the diaphragm (which in this case confirmed bilateral diaphragmatic paralysis), may show paradoxical movement of the lateral part of the relevant dome on sniffing. ${ }^{1}$ Transdiaphragmatic pressures may also be assessed during maximum static inspiratory effort. Electromyography may show denervation of cervically innervated muscles. Magnetic resonance imaging of cervical spine may demonstrate evidence of cord compression.

\section{QUESTION 3}

Therapy includes deep breathing exercises, incentive spirometry (which acts by increasing the work of the accessory muscles), and nasal continuous positive-pressure ventilation, ${ }^{2}$ the increased expiratory pressure reducing the paradoxical movement of the diaphragm during subsequent inspiration, thereby increasing diaphragmatic efficiency. More aggressive

1 Robotham JL, Chipps BE, Shermeta DW. Continuous positive airway pressure in hemidiaphragmatic paralysis. Anaesthesiology 1980; 52: 167-70.

2 Miller J, Moxham J, Green M. The maximal sniff in the assessment of diaphragm function in man. Clin Sci 1985; 69: assessm.

\section{Causes of diaphragmatic paralysis}

- trauma

- cardiac surgery (particularly in children), where injury to the phrenic nerve may occur during retraction of the pericardium

- neck manipulation and cervical disc surgery

- neurological disorders: demyelinating disease, muscular dystrophies, myopathies and neuralgic amyotrophy

treatment options include surgical plication ${ }^{3}$ of the diaphragm (where the diaphragm is folded on itself and pleated, in order to increase its tensile strength). Alternatively, the diaphragm may be 'paced' by implantation of diaphragmatic pacemakers. ${ }^{4}$

In the current case, incentive spirometry and deep breathing exercises led to a progressive improvement in both symptoms and lung volumes over an eight-month period. Unfortunately, a trial of nasal continuous positive pressure ventilation was not tolerated. At present our patient complains only of persistent orthopnoea, and has had further recovery of pulmonary function $\left(\mathrm{FEV}_{1} 1.51 \mathrm{l}\right.$, FVC $1.91 \mathrm{l})$.

\section{Final diagnosis}

Post-traumatic bilateral diaphragmatic palsy.

Keywords: orthopnoea, diaphragmatic paralysis

3 Ciccolella DE, Daly BT, Celli BR. Improved diaphragmatic function after surgical plication for unilateral diaphragmatic paralysis. Am Rev Respir Dis 1992; 146: 797-9.

4 Elefteriades JA, Hogan JF, Handler A, Loke JS. Long term follow-up of bilateral pacing of the diaphragm in

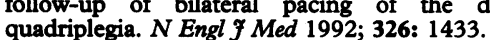

